OPEN ACCESS

Edited by:

Antonio Politano,

University of L'Aquila, Italy

Reviewed by:

Chong Leong Gan,

Micron, United States Bumho Kim,

University of Pennsylvania,

United States

${ }^{*}$ Correspondence:

Libo Gao

Ibgao@xidian.edu.cn

Jibao Lu

jibao.lu@siat.ac.cn

Rong Sun

rong.sun@siat.ac.cn

Specialty section:

This article was submitted to

Thin Solid Films,

a section of the journal

Frontiers in Materials

Received: 01 September 2021

Accepted: 01 November 2021

Published: 24 November 2021

Citation:

Wu L, Wang W, Zhao H, Gao L, Lu J and Sun $R$ (2021) A Molecular

Dynamics Simulation Study: The Inkjet Printing of Graphene Inks on

Polyimide Substrates.

Front. Mater. 8:769071.

doi: 10.3389/fmats.2021.769071

\section{A Molecular Dynamics Simulation Study: The Inkjet Printing of Graphene Inks on Polyimide Substrates}

\author{
Lingjun Wu ${ }^{1}$, Wei Wang ${ }^{1}$, Haitao Zhao $^{2}$, Libo Gao ${ }^{3,4 *}$, Jibao $\mathrm{Lu}^{1 *}$ and Rong Sun ${ }^{1 *}$ \\ ${ }^{1}$ Shenzhen Institute of Advanced Electronic Materials, Shenzhen Institute of Advanced Technology, Chinese Academy of \\ Sciences, Shenzhen, China, ${ }^{2}$ Materials Interfaces Center, Shenzhen Institutes of Advanced Technology, Chinese Academy of \\ Sciences, Shenzhen, China, ${ }^{3}$ School of Mechano-Electronic Engineering, Xidian University, Xian, China, ${ }^{4}$ CityU-Xidian Joint \\ Laboratory of Micro/Nano-Manufacturing, Shenzhen, China
}

Inkjet printing-based 2D materials for flexible electronics have aroused much interest due to their highly low-cost customization and manufacturing resolution. However, there is a lack of investigation and essential understanding of the surface adhesion affected by the printing parameters at the atomic scale. Herein, we conducted a systematic molecular dynamics simulation investigating the inkjet printing of graphitic inks on polyimide substrates under various conditions. Simulations under different temperatures, inkjet velocities, and mechanical loadings such as pressure and deformation are performed. The results show that the best adhesion is achieved in the plasma-modified polyimide/ graphene-oxide $(\mathrm{mPI} / \mathrm{GO})$ interfacial system (the interaction energy $\left(\mathrm{E}_{\mathrm{in}}\right)$ between $\mathrm{mPI}$ and $\mathrm{GO}$ is ca. 1.2 times than with graphene). The adhesion strength decreases with increasing temperature, and higher inkjet velocities lead to both larger impact force as well as interfacial fluctuation, while the latter may result in greater interfacial instability. When loaded with pressure, the adhesion strength reaches a threshold without further improvement as continuing compacting of polymer slabs can hardly be achieved. The detachment of the interfaces was also explored and $\mathrm{mPI} / \mathrm{GO}$ shows better resistance against delamination. Hopefully, our simulation study paves the way for future inkjet printing-based manufacturing of graphene-based flexible electronics.

Keywords: surface adhesion, inkjet printing, 2D material, graphene, polyimide, molecular dynamics

\section{INTRODUCTION}

Flexible electronics are attracting increasing attention nowadays due to various superior properties over traditional rigid electronics. These bendable and even foldable apparatus are much reduced in weight compared to metal-based electronics. Organic and polymeric substrates are widely used in manufacturing, and in general, the potential producing cost is much lower. To this day, flexible electronics is witnessed to have growing applications in various fields, such as sensors (Sekitani et al., 2009; Gao et al., 2019; Xu et al., 2020; Gao et al., 2021a; Gao et al., 2021b; Gao et al. 2020; Huang et al., 2014; Lu et al., 2021; Li et al., 2021), electronic displays (Templier et al., 2007; Mizukami et al., 2018; Zhou et al., 2006; Han et al., 2012), solar cells (Granqvist 2007; Peng et al., 2017), nanogenerators (Liu et al., 2021), transistors (Chung et al., 2019), etc. Polyimides (PI) are one of the most widely polymeric flexible substrates used (Berggren et al., 2007; Lien et al., 2014; Song et al., 2017) due to their excellent flexibility and outstanding thermal stability $\left(\mathrm{T}_{\mathrm{g}}: \mathrm{ca} \cdot 400^{\circ} \mathrm{C}\right)$, as well as being chemically inert under various conditions. 
In the pattern deposition of flexible electronics, inkjet printing using graphitic inks, such as pristine graphene $(\mathrm{PG})$ or graphene oxide (GO) is a promising direction (Song et al., 2017; Li et al., 2013, 2017; Fang et al., 2016a). Inkjet printing technique surpasses other deposition methods in many ways, such as its high printing resolution, fast printing speed, relatively lower cost, less production of waste, as well as its much-simplified operation procedures (Fang et al., 2016b; Moses et al., 2020), thus has been widely applied in semiconductor electronics (Noguchi et al., 2008; Minemawari et al., 2011; Kim et al., 2016). The PG/GO inks are receiving growing attention these days due to two main advantages: first, their electric conductivity is comparable to the metal-based inks, while much higher than the conductive polymers (Moses et al., 2020; Saidina et al., 2019). Besides, they are more compatible with the polymeric substrates used in flexible electronics, as they are light in weight and also chemically and mechanically stable (He et al., 2019). To improve the sensitivity of the circuits and achieve better performance, a snugly combined, well-fabricated ink/substrate interface is highly desired. The inkjet fabrication process can have a significant impact on the ink/substrate interfacial adhesion, such as the substrate temperature, the inkjet velocity, etc. Meanwhile, flexible electronics are usually applied at various working conditions, e.g., they are often exerted under different types of loadings, such as bending, stretching, pressing, etc. Therefore, it is also crucial that a good interfacial adhesion is guaranteed under different loadings to improve the service life and work stability.

Therefore, understanding the interfacial properties between the ink and the substrate is critical in the improvement of flexible electronics. Though the macroscopic properties have been well characterized via experimental techniques, such as scanning electron microscope (SEM), transmission electron microscope (TEM) (Lai et al., 2021), and mechanical tests (Xu et al., 2020), nonetheless information regarding micro-scale properties at the atomic level, especially dynamic changes on an ultrafast timescale, are still difficult to obtain via experiments. Simulation will be an ideal complementary means to experimental techniques regarding microscale and fast-timescale probes. Molecular dynamics (MD) simulation is a powerful tool in investigating the physical properties of materials at the atomic scale. While comparing to the first-principle calculations, for which the simulation scale is limited to hundreds of atoms, the simulation system of MD can reach as large as $10^{4}-10^{6}$ atoms with well simulation accuracy. Moreover, ultrafast-timescale simulations down from fs to $\mu$ s can be achieved using MD. MD simulations have widely been used in investigating the interfacial properties of composite materials (Faasen et al., 2020; Jarray et al., 2020). Patil et al. (2020) conducted an MD investigation on the interfacial properties between flattened carbon-nanotube $(\mathrm{CNT})$ and polyimides. Their results suggest weaker interfacial interactions between fluorinated polyimide and CNT. Besides, both the friction and tensile process of the PI/CNT interface are studied systematically using MD with a more comprehensive understanding of the failure mechanism. Liu et al. (2015) used MD to investigate the pulling out of the graphene sheet in the composite and the change of the polymer/graphene interfacial properties during the process. The morphology of the graphene sheet, polymer types, and chain length are found to have a crucial impact on the mechanical strength of the interface. Though these MD studies have offered crucial insight into a wide range of interfacial problems, however, to the best of our knowledge, MD investigation on the graphitic ink/polyimide substrate interfacial behaviors during inkjet printing and deformation in the application have rarely been reported. A deeper theoretical exploration is desired for future technical improvements.

In this study, a systematic MD simulation investigation on the inkjet printing of graphitic inks on polyimide substrates has been conducted. The plasma treatment of the PI substrate is examined with two types of graphitic inks, PG and GO. First, the effect of temperature and inkjet velocity during inkjet printing are investigated. Second, the interfacial deformation behaviors under both downward pressure and delamination are examined for the evaluation of working performance. The organization of the paper is as follows: Section 2 is the computational methods used in this work, Section $\mathbf{3}$ is the results and discussions of the simulations, and Section 4 is the conclusion. The goal of our work is that the simulation results can be helpful and instructive for future technical improvements in the inkjet printing-based fabrication of flexible circuits.

\section{MODELS AND METHODS}

\subsection{Force Field}

The Scienomics' Polymer Consistent Force Field (SciPCFF) is used in describing the interaction in all systems. SciPCFF is developed by Scienomics based on the polymer consistent force field (PCFF) with extended parameters from the condensed-phase optimized molecular potentials for atomistic simulation force field (COMPASS). Both PCFF and COMPASS force fields have been widely used in the simulation of polymeric and graphitic materials (Yang et al., 2013; Chawla and Sharma 2017). Lennard-Jones (9-6) potential is used in describing the van der Waals interaction.

\subsection{Computational Details}

The Large-scale Atomic/Molecular Massively Parallel Simulator (LAMMPS) program is used for MD simulations (Plimpton 1995). The atomic trajectory is integrated using the velocity Verlet algorithm. Timestep of $1 \mathrm{fs}$ is used. All electrostatic interactions are calculated using the Coulombic terms with the reciprocal-space contribution calculated using the particle-particleparticle-mesh (pppm) method. A Nóse-Hoover thermostat and barostat are used in controlling the temperature and pressure, respectively. Periodic boundary conditions are applied in all directions. In simulations where no periodicity in the $\mathrm{z}$-direction is wanted, the PBC in the $\mathrm{z}$-direction is canceled and a large vacuum space is set in the $\mathrm{z}$-direction.

\subsection{Building the Simulation Systems \\ 2.3.1 Polymers}

The initial polymer structures are built using the Amorphous Builder module in the Materials and Processes Simulations 
(MAPS) software developed by Scienomics SARL. Both Polymer bulks of poly $\left(4,4^{\prime}\right.$-oxydiphenylene-pyromellitimide) (PI) and plasma-modified PI (mPI) are built, the corresponding chemical formulas are illustrated in Supplementary Figure S1. A chain length of 10 repeating units is used in both cases. First, the polymer bulks are compressed under a pressure of $1 \mathrm{~atm}$ at $1,000 \mathrm{~K}$ for $2 \mathrm{~ns}$, reaching a density at $1.351 \mathrm{~g} / \mathrm{cm}^{3}$ and $1.346 \mathrm{~g} /$ $\mathrm{cm}^{3}$ for PI and $\mathrm{mPI}$, respectively, which is close to the experimental value of PI at $1.42 \mathrm{~g} / \mathrm{cm}^{3}$. Then an annealing procedure is conducted under canonical ensemble (NVT) from 1,000 to $300 \mathrm{~K}$ for $7 \mathrm{~ns}$, and at last, the systems are equilibrated at $300 \mathrm{~K}$ for $500 \mathrm{ps}$. The $\mathrm{x}-\mathrm{y}$ dimensions of the final polymer bulks are ca. $90 \times 90 \AA^{2}$.

\subsubsection{PG and GO}

The structure of PG is taken from the structure library of MAPS, while GO is built using the open-source Python tool GOPY (Muraru et al., 2020) with PG as the initial configuration. To match the dimension of the polymer bulks, a PG of $90 \times 90 \AA^{2}$ is constructed. An oxidation ratio of $22.5 \%$ is used for GO. The formula of GO is $\mathrm{C}_{9}(\mathrm{OH})_{1}(\mathrm{O})_{1}$, which means the ratio of carbon, hydroxyl groups, and epoxy groups is 9:1:1. Using GOPY, these functional groups are randomly placed on the PG sheet on both sides. The snapshot of the GO structure is demonstrated in Supplementary Figure S2.

\subsubsection{Interface}

Using MAPS four interface models are built (PI/PG, PI/GO, mPI/ $\mathrm{PG}$, and $\mathrm{mPI} / \mathrm{GO}$ ) by combing the polymer bulks and interfaces as mentioned before. To reach a better adhesion, a compress procedure is conducted. The ink parts are fixed as rigid during the whole simulation. Firstly, the systems are heated from $300 \mathrm{~K}$ to $1,000 \mathrm{~K}$ with a force of $0.005 \mathrm{Kcal} \mathrm{mol}^{-1} \AA^{-1}$ imposed on the polymer slab towards the ink. Then the temperature is kept at $1,000 \mathrm{~K}$ with the force on for $200 \mathrm{ps}$. Next, the temperature is gradually reduced to $300 \mathrm{~K}$ with the loading gradually decreasing to zero. Finally, the whole system is equilibrated for another 200 ps. The final structure reaches an ideal attachment between the ink and the substrate, as illustrated in Supplementary Figure S3.

\section{RESULTS AND DISCUSSION}

\subsection{Ink/Substrate Interface During Inkjet Printing-Effects of Temperature}

One problem often encountered in inkjet printing is the adhesion strength of the ink/substrate interface. Stronger adhesion is favored for better electric performance as it helps improve the electric conduction of the circuits. Reduced-graphene oxide (rGO) inks are among the most commonly used graphitic inks. rGO is hydrophilic as normally no absolute reduction of GO can be achieved. However, as PI is a relatively hydrophobic material, usually its surface needs further modification to improve its adhesion with the rGO inks. Plasma treatment has been widely used as one of the most popular among many surface modification methods (Kondo et al., 2017; Rusu et al., 2020;
Marques et al., 2019; Abdel-Fattah and Alshaer, 2020; Usami et al., 2012; Park et al., 2010; Chan et al., 1996; Least and Willis 2013; Ghosh et al., 1997; Huang et al., 2003; Shin and Kang, 2010) due to various advantages such as high efficiency, rapid and simple operation, no need for solvents, and the ability to introduce a wide range of functional groups onto the surface (Abdel-Fattah and Alshaer, 2020). Meanwhile, after plasma treatment, only the very surface of the polymer is etched without significantly changing the properties of the inner bulk (Abdel-Fattah 2019; Park et al., 2009; Xie et al., 2011). Based on previous research, the imide bond $\mathrm{N}-\mathrm{C}$ in $\mathrm{PI}$ is proposed to be fairly weak and gets easily cleaved, forming amide and carboxylate groups under various conditions. This mechanism has been observed under the treatment of acid (Ghosh et al., 1997), base (Huang et al., 2003), UV irradiation (Rusu et al., 2020), and plasma (Zhou et al., 2019; Grandoni et al., 2017). Inagaki et al. investigated the modification of $\mathrm{PI}$ using seven different plasmas: Ar-, $\mathrm{N}_{2^{-}}, \mathrm{O}_{2^{-}}, \mathrm{CO}_{2^{-}}, \mathrm{NO}-$, and $\mathrm{NO}_{2}$-plasmas. The results show that these different types of plasma all lead to the cleavage of the $\mathrm{N}-\mathrm{C}$ bond in the imide group as well as the following formation of secondary amide and carboxylate groups (Inagaki et al., 1992), as shown in Supplementary Figure S1. Therefore, we use the formula in Supplementary Figure S1 as the structure of modified PI (mPI).

However, the micro-scale mechanism of the improved adhesion after plasma treatment still needs a more thorough investigation for $2 \mathrm{D}$ materials. Here we use $\mathrm{MD}$ to explore the adhesion between two types of graphitic inks, PG and GO, with two substrates, the pristine PI and plasma-modified PI (mPI), in an attempt to explore the effects of graphitic inks and the surfacemodification of PI on the adhesion strength. The detailed building process of models can be found in Section2.3. Here the adhesion under different temperatures is studied as the substrate's temperature is a crucial parameter affecting the printing result of the inkjet printing process. The temperature control and related simulation details during calculation are described in Section 2.2. The adhesion strength is evaluated via the calculation of the interaction energy $\left(\mathrm{E}_{\mathrm{in}}\right)$ between the polymer slab and the GO/PG ink, which is the reduced potential energy after the separated components combine with each other, as clarified as follow:

$$
E_{\text {in }}=E_{\text {tot }}-\left(E_{\text {ink }}+E_{\text {substrate }}\right)
$$

$E_{\text {tot }}, E_{\text {ink }}$ and $E_{\text {substrate }}$ are the potential energies of the composite, ink and substrate, respectively. A lower $E_{\text {in }}$ indicates stronger interfacial adhesion strength. The results are shown in Figure 1A.

First of all, one can observe in Figure 1A that under same temperatures the adhesion strength follows: PG-mPI $<$ PG-PI $<$ $\mathrm{GO}-\mathrm{PI}<\mathrm{GO}-\mathrm{mPI}$. The adhesion of polymeric substrates with GO is in general greater than that with PG. This is attributed to the fact that both the van der Waals (vdW) and the Coulomb electrostatic interactions exist between the polymers and GO, including the strong hydrogen bonding interactions, while with PG only the vdW interaction is present. The enhanced adhesion between $\mathrm{mPI}$ and GO is consistent with the experimental observation that PI surface turns more hydrophilic after 

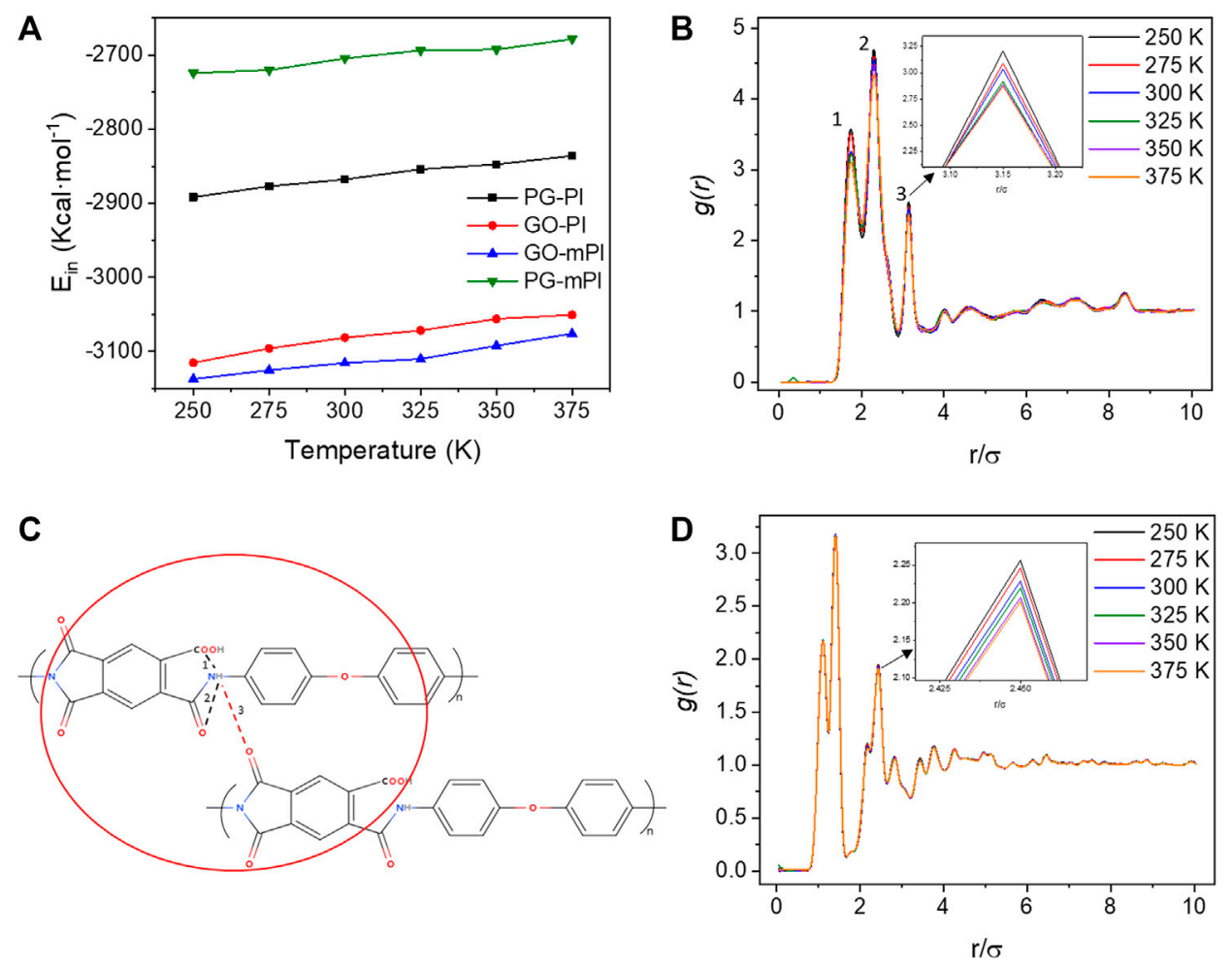

FIGURE 1 | (A) The interaction energies $\mathrm{E}_{\text {in }}$ under different temperatures; (B) NH-CO RDF of $\mathrm{mPI} / \mathrm{GO}$ under different temperatures, inset graph: amplified peak 3 ; (C) Scheme of peaks 1, 2, 3; (D) all-to-all RDF of mPl/GO.

plasma treatment, and the adhesion of rGO ink is much improved. This phenomenon is mainly attributed to the enhanced interfacial hydrogen bonding interactions. E.g., in $\mathrm{mPI} / \mathrm{GO}$, both $\mathrm{COOH}$ and $\mathrm{NH}$, as well as $\mathrm{OH}$ groups on $\mathrm{GO}$ can donate their $\mathrm{H}$ in forming interfacial hydrogen bonds, while in $\mathrm{GO} / \mathrm{PI}$, only $\mathrm{OH}$ groups on $\mathrm{GO}$ can act as the hydrogen donors. The weakest adhesion is observed between PG and $\mathrm{mPI}$, of which the difference in polarity is the greatest among all systems. The results indicate that though GO is more adhesive on $\mathrm{mPI}$, if using PG as ink, unmodified PI will show better adhesion.

A lower temperature is found to help the improvement of adhesion strength for all systems, as reflected via the proportional relationship between $\mathrm{E}_{\text {in }}$ and temperature in the range investigated. This is mainly attributed to two reasons: first, the non-bonding interactions (vdW, hydrogen bonding, and other electrostatic interactions) are more unstable under higher temperatures. This can be viewed via the radial distribution functions (RDF). RDF describes the density of a specific particle $B$ as a function of distance from a reference particle A, reflecting the possibility of finding $B$ around A. $r / \sigma$ is distance $r$ divide the distance unit ( $\mathrm{A}$ in this case). $\mathrm{g}(\mathrm{r})$ represents the density of the specific particle in the micro-shell defined by $r$ relative to the entire system. As aforementioned, the amide and carboxylate groups formed after cleavage of N-C bond as the result of plasma treatment is the main cause of the improved adhesion of mPI. Therefore, to explore the impact of temperature on the pair interaction between the amide hydrogens $(\mathrm{NH})$ with the carbonyl oxygens (CO), the corresponding RDF under different temperatures is analyzed. Here the $\mathrm{mPI} / \mathrm{GO}$ system is presented (RDF of mPI/PG is shown in Supplementary Figure S5) as illustrated in Figure 1B. Peaks 1, 2, and 3 correspond to the intra-molecular hydrogen bond between $\mathrm{NH}$ and the carboxylate oxygen (COO), the interaction between $\mathrm{NH}$ and the oxygen of the bonded carbonyl, and the inter-molecular $\mathrm{NH}-\mathrm{CO}$ hydrogen bonds, respectively, as illustrated in Figure 1C. The magnified peak 3 is shown in the inset in Figure 1B, and peaks 1, 2 can be found in the Supplementary Figure S4. One can observe that all three peaks drop in intensity with increasing temperature, reflecting the instability of these interactions upon heating. A similar result is also observed for other non-bonding interactions, as reflected by the all-to-all RDF shown in Figure 1D (the all-to-all RDF of other systems is shown in Supplementary Figures S5D, S6). The first two peaks within $1.5 \AA$ are considered to be the covalent bonds, while the peak at ca. $2.4 \AA$ magnified in the inset is assigned to the overall nonbonding interactions, which drop in intensity with increasing temperature. Second, as higher temperature generally results in lower density of the system, the overall non-bonding interactions near the interface are also reduced.

\subsection{Ink/Substrate Interface During Inkjet Printing-Effects of Inkjet Velocity}

As discussed before, the mPI/GO system shows the best adhesion performance due to enhanced hydrophilicity after plasma 

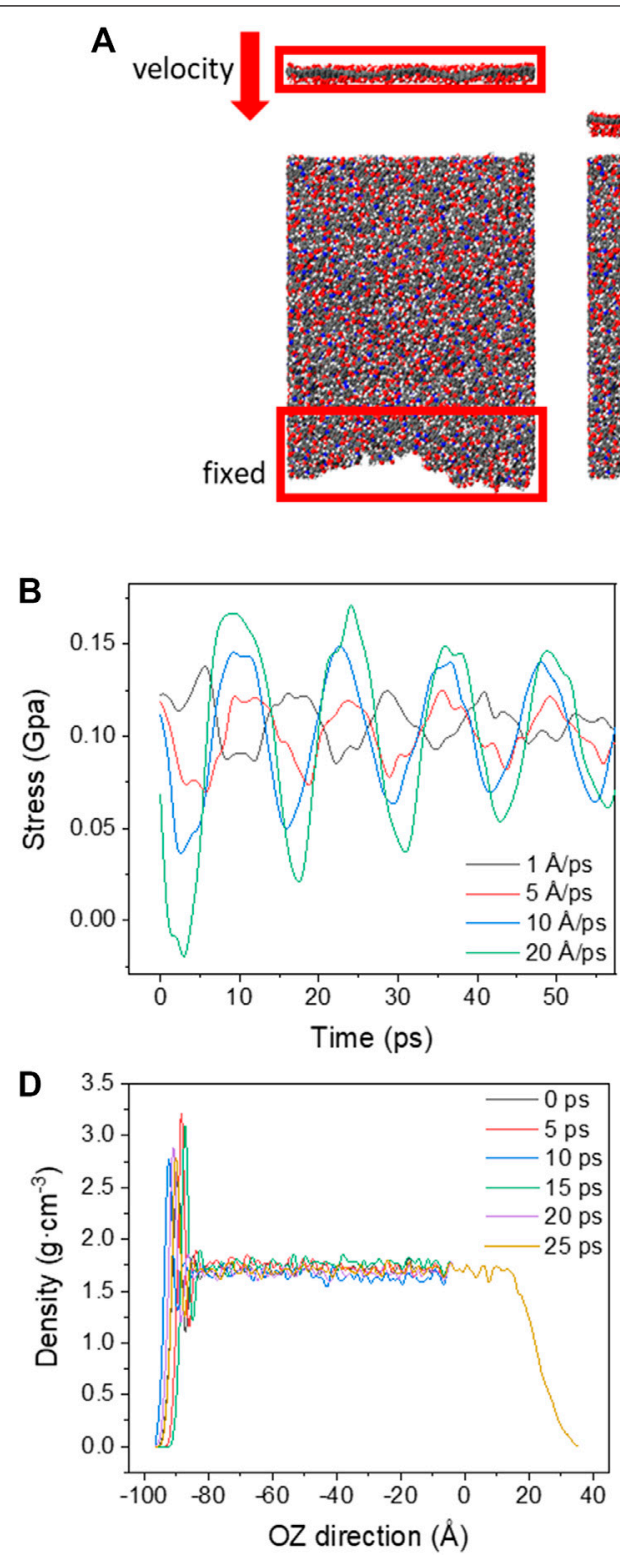
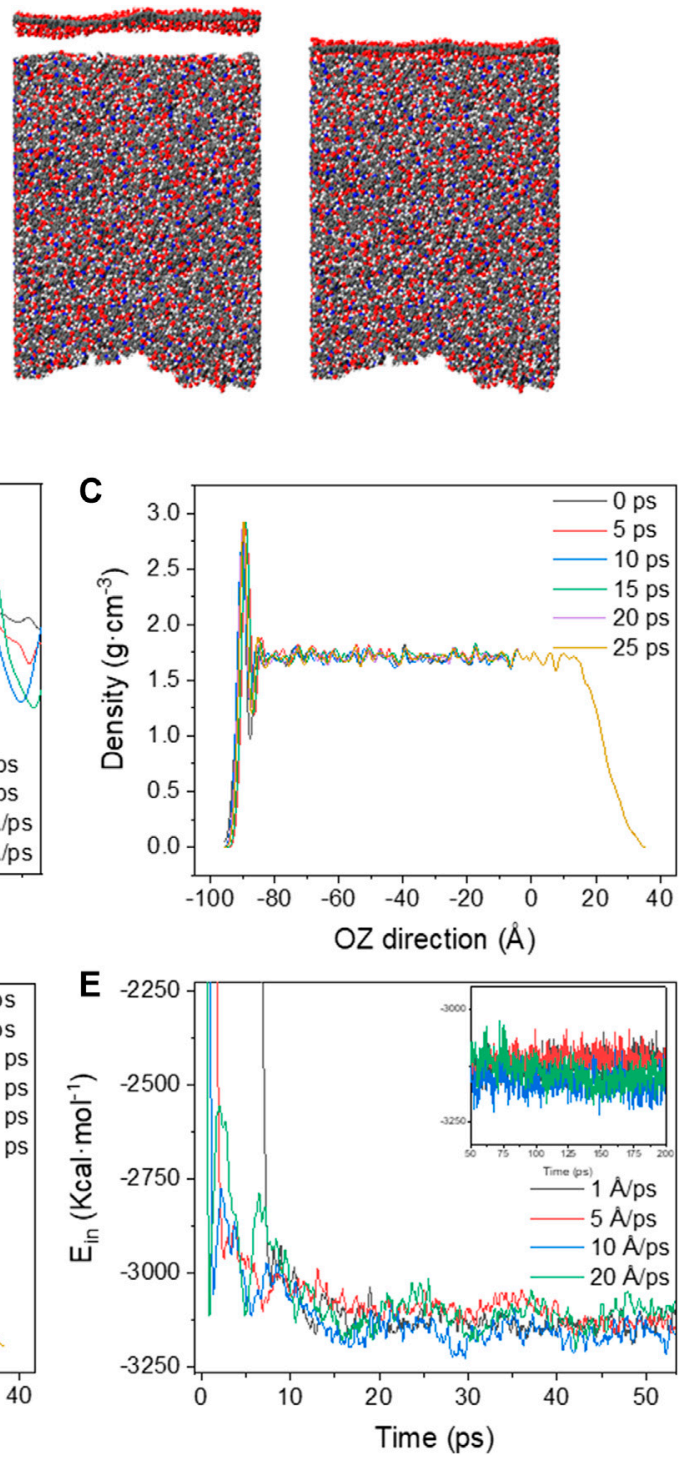

FIGURE 2 | (A) Scheme of shooting GO onto mPI; (B) stress of mPl/GO upon hitting; density profiles of mPl/GO system under (C) $1 \AA$ Aps, d) $10 \AA$ ps; (E) $\mathrm{E}_{\text {in }}$ change of $\mathrm{mPl} / \mathrm{GO}$ system under different inkjet velocities upon hitting; inset: magnified $\mathrm{E}_{\text {in }}$ change from 50 to 200 ps.

treatment. In fact, in real applications, mPI has already replaced $\mathrm{PI}$ as the substrates in flexible electronics. Therefore, we will focus the investigation on mPI systems. Apart from temperature, we are also interested in how the interface behaves under different inkjet velocities. Four velocities $(1,5,10$, and $20 \AA / \mathrm{ps})$ are investigated. Nonetheless, it has to be pointed out that due to the consideration of computational cost, the simulated inkjet velocities are much higher in comparison to the ones used in real applications $(c a .5 \times$ $\left.10^{-4} \AA / \mathrm{ps}\right)$. The bottom part of the polymer substrate is set as rigid, while the ink (PG/GO) is given an initial velocity towards the substrate in the $\mathrm{z}$-direction, as demonstrated in Figure 2A. It is found that the ink/substrate interface is affected by the inkjet velocity in two aspects. Faster printing velocity leads to a larger impact force of ink onto the substrate, and will also result in greater interfacial fluctuation. The impact force can be reflected via the amplitude of the stress fluctuation, as shown in Figure 2B; Supplementary Figure S7A. This can also be observed via the density profiles under different velocities (Figures 2C,D; Supplementary Figures S7B,C). As shown in Figure 2C; Supplementary Figure S7B, under slow velocities ( $1 \AA / \mathrm{ps})$, the density near the interface ( -90 to $-80 \AA$ ) is relatively stable with the gap between the ink and the substrate clearly shown at -87.66 and $-85.77 \AA$ for systems mPI/GO and mPI/PG, respectively. While under higher velocities (Figure 2D; Supplementary Figure S7C, $10 \AA / \mathrm{ps}$ ), the density fluctuates rather vigorously, especially near the interface, and the ink/substrate gap can no 

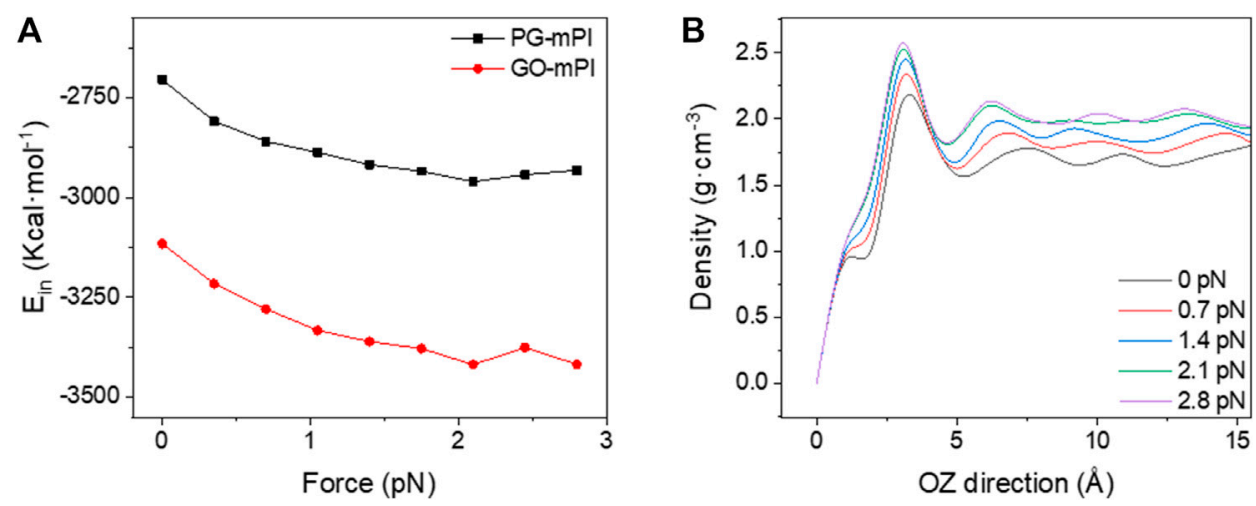

FIGURE 3 | (A) the interaction energies $\mathrm{E}_{\text {in }}$ under different pressures; (B) density profiles of mPI/GO under different pressures.

longer be observed. And the combined influence of impact force and interfacial fluctuation affects the interfacial adhesion, as reflected by $\mathrm{E}_{\text {in }}$ (Figure 2E; Supplementary Figure S7D). Higher inkjet velocity, in general, leads to greater fluctuation of $\mathrm{E}_{\text {in }}$ after hitting. Though higher inkjet velocity leads to a larger impact force, the greater interfacial fluctuation may also have a significant influence on the ink's adhesion. And as observed in the inset in Figure 2E, after ca. 100 ps $\mathrm{E}_{\text {in }}$ is basically stabled. For $\mathrm{mPI} / \mathrm{GO}$, after $100 \mathrm{ps}, \mathrm{E}_{\text {in }}$ under higher inkjet velocities (10 and $20 \AA / \mathrm{ps}$ ) is lower compared to lower velocities ( 1 and $5 \AA / \mathrm{ps}$ ), indicating that the interfacial adhesion between $\mathrm{mPI}$ and GO is enhanced by higher inkjet velocity. While for $\mathrm{mPI} / \mathrm{PG}$, there is no significant difference between different velocities. Therefore, to achieve better adhesion via tuning inkjet velocities, both impact force, as well as interfacial fluctuation, should be considered.

\subsection{Ink/Substrate Interface Under Loading-Downward Pressure}

Another issue often encountered in flexible electronics is that the flexible circuit is often under different types of loading during use, such as downward pressing, bending, stretching, detaching, etc. Therefore, understanding how the interface is affected under loading is critical in achieving better working performance. Here we have analyzed two types of loading, the downward pressing and the detachment under uniaxial tensile. In this section, the effects of downward pressing are firstly discussed. In many pressure-sensitive flexible electronics, the conduction and sensitivity of the circuits are highly related to the pressure applied. The pressure change in the electronics can be captured as electric signals. Therefore, it is crucial to understand how the interface is affected by pressures, which is the key to improving the sensitivity of the electronics. The results are shown in Figure 3. $\mathrm{E}_{\text {in }}$ of different systems under different pressures at $300 \mathrm{~K}$ are calculated (in each case forces $0,0.35,0.7$, $1.05,1.4,1.75,2.1,2.45$, and $2.8 \mathrm{pN}$ are applied on each atom in the mPI slab downward, while PG/GO is fixed).

As the force applied on the atom increases from 0 to $1.75 \mathrm{pN}$, the adhesion is significantly improved as reflected by the drop of $\mathrm{E}_{\text {in }}$ (Figure 3A). However, the adhesion strength can only be improved through increasing the pressure to a limited extent, as it can be observed in Figure 3A that a further increment of force to $2.8 \mathrm{pN}$ results in a much smaller change in $\mathrm{E}_{\mathrm{in}}$. The reason is mainly attributed to the interfacial density $\left(\rho_{\text {in }}\right)$ change under different pressures, as demonstrated in the density profiles near the interface as illustrated in Figure 3B; Supplementary Figure S8. Higher density leads to a denser formation of non-bonding interactions and stronger adhesion strength in the interfacial regions. The changes in $\rho_{\text {in }}$ are consistent with $\mathrm{E}_{\text {in }} \cdot \rho_{\text {in }}$ gradually increases with compression at first, however with further pressure increases, the increase of $\rho_{\text {in }}$ is relatively small, and significant enhancement in compaction can no longer be achieved. The results indicate that the interfacial adhesion can only be improved via increasing the pressure to a certain extent, which may help in the improvement of the electronics' sensitivity.

\subsection{Ink/Substrate Interface Under Loading-Detachment During Tensile}

As mentioned above, in flexible electronics, the ink/substrate interface is usually subjected to different types of external loading. In real applications, except the normal pressure or impact downwards, printed ink on flexible substrates may also suffer in the delamination off the interface. Here the investigation of the detachment process of the mPI systems has been conducted by applying uniaxial tensile loading.

The upper part of the polymer bulk is fixed, while the PG/GO layer is given a constant velocity downwards ( $1 \AA / \mathrm{ps})$, as shown in the snapshot at time 0 ps in Figure 4A. The movable part of the polymer slab is kept at the thickness of $90 \AA$ for both systems.

As shown in the snapshots in Figure 4A (The snapshots for $\mathrm{mPI} / \mathrm{PG}$ are shown in Supplementary Figure S9), which can intuitively demonstrate the change during simulation, the downward movement of the ink is accompanied by the concurrent movement of the polymer chains attached to it due to interfacial adhesion. As reflected in the corresponding snapshots at different times for both systems, at the initial stage upon tensile $(10 \mathrm{ps})$ the polymer slabs are stretched without apparent formation of micro-voids. Then along the process, the micro-voids within the polymer bulk witnesses a 


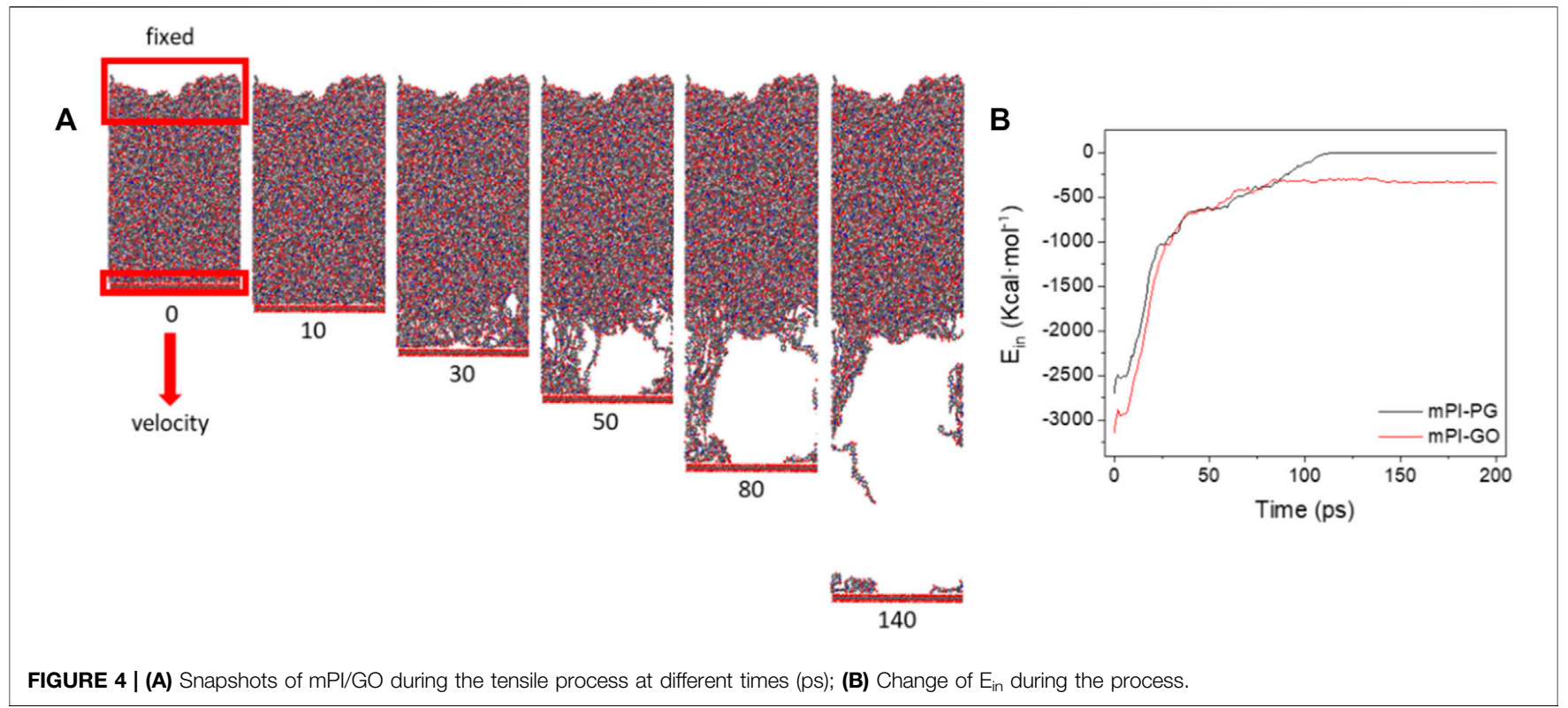

growth of size. The chains are separated from each other, and the ones near the surface are then detached. As shown in the last snapshots for both systems, the final detachment for $\mathrm{mPI} / \mathrm{GO}$ occurs at a time of $140 \mathrm{ps}$ with chain residues at the interface, while for $\mathrm{mPI} / \mathrm{PG}$, complete detachment occurs earlier, at $120 \mathrm{ps}$. The detachment can also be viewed via the $\mathrm{E}_{\text {in }}$ change as shown in Figure 4B. For both systems, $\mathrm{E}_{\text {in }}$ increases with the gradual interfacial detachment of chains. The most drastic increase of $\mathrm{E}_{\text {in }}$ is observed before $c a .30 \mathrm{ps}$, as in this stage, most chains are still attached to the interface. After this stage, as many chains are pulled away from the interface, the corresponding change of $E_{\text {in }}$ also gets much slower, as more clearly illustrated in the snapshots at 50 and $80 \mathrm{ps}$. The complete detachment of $\mathrm{mPI} / \mathrm{PG}$ is reflected by the $E_{\text {in }}$ increase to 0 at the end, and for mPI/GO the chain residue on the interface is evaluated by the final $\mathrm{E}_{\text {in }}$ at $c a$. $-337 \mathrm{kcal} / \mathrm{mol}$. As shown in Figure 4B, though the final detachment for mPI/GO occurs at $140 \mathrm{ps}$, however after $c a$. 90 ps no apparent change of $E_{\text {in }}$ is observed. This indicates that the chain residues are firmly attached to the interface, and the final fracture actually occurs between the chain residues on the interface and the ones in the polymer slab instead of exact failure at the surface. These results are ascribed to the stronger adhesion strength between $\mathrm{MPI}$ and GO. The results indicate that $\mathrm{mPI} / \mathrm{GO}$ system shows better resistance against uniaxial detachment due to greater interfacial adhesion strength.

\section{CONCLUSION}

In this study, a systematic MD investigation focusing on graphitic ink/PI substrate inkjet printing has been conducted. The ink/substrate adhesion under different temperatures, the impact of inkjet velocities during the printing process, the interfacial behaviors of the mPI systems under different loading have been explored at the atomic level on an ultrafast timescale. $\mathrm{MD}$ results suggest the adhesion strength is follows: $\mathrm{PG}-\mathrm{mPI}<\mathrm{PG}-\mathrm{PI}<\mathrm{GO}-\mathrm{PI}<$ GO-mPI, with the best adhesion strength achieved between $\mathrm{mPI}$ and GO, as it improved after introduction of hydrophilic groups through plasma treatment, which is consistent with the experimental results. However, unmodified PI is found to have better adhesion with the PG ink. The ink/substrate adhesion decreases with increasing temperature due to the instability of non-bonding interactions as well as expanded systems at higher temperatures. Investigation on the inkjet velocities of $\mathrm{mPI}$ systems suggests that higher inkjet velocity leads to both greater impact force as well as more drastic interfacial fluctuation. When the systems are under different pressures, the interfacial adhesion is at first significantly enhanced, which is attributed to the increased density near the interface. However, further increases in pressure helps little in the improvement of adhesion strength. Tensile simulation suggests delamination occurs in the interface under uniaxial loading. The detachment along tensile is accompanied with the formation and growth of micro-voids. For mPI/GO, the greater interfacial adhesion leads to the residue of chains on the interface as well as the later occurrence of failure. In conclusion, the simulation results in this work have provided theoretical views on the interfacial behaviors in graphitic ink/PI substrate systems at the atomic level during inkjet printing as well as in applications, which may guide future technical developments and improvements.

\section{DATA AVAILABILITY STATEMENT}

The original contributions presented in the study are included in the article/Supplementary Material, further inquiries can be directed to the corresponding authors. 


\section{AUTHOR CONTRIBUTIONS}

All authors listed have made a substantial, direct, and intellectual contribution to the work and approved it for publication.

\section{FUNDING}

This work was supported by the National Natural Science Foundation of China (no. 52003289, 52173234), the Shenzhen-Hong Kong-Macau Technology Research Program (Type C, 202011033000145), the Natural Science Foundation of Shaanxi Province (No.2020JQ-295), the Fundamental

\section{REFERENCES}

Abdel-Fattah, E. (2019). Surface and Thermal Characteristics Relationship of Atmospheric Pressure Plasma Treated Natural Luffa Fibers. Eur. Phys. J. D. 73 (4), 71. doi:10.1140/epjd/e2019-90281-3

Abdel-Fattah, E., and Alshaer, M. (2020). Polyimide Surface Modification Using He-H2o Atmospheric Pressure Plasma Jet-Discharge Power Effect. Coatings. 10 (7), 662. doi:10.3390/coatings10070662

Berggren, M., Nilsson, D., and Robinson, N. D. (2007). Organic Materials for Printed Electronics. Nat. Mater. 6 (1), 3-5. doi:10.1038/nmat1817

Chan, C. -M., Ko, T. -M., and Hiraoka, H. (1996). Polymer Surface Modification by Plasmas and Photons. Surf. Sci. Rep. 24 (1), 1-54. doi:10.1016/0167-5729(96) 80003-3

Chawla, R., and Sharma, S. (2017). Molecular Dynamics Simulation of Carbon Nanotube Pull-Out From Polyethylene Matrix. Composites Sci. Technology. 144 (May), 169-177. doi:10.1016/j.compscitech.2017.03.029

Chung, S., Cho, K., and Lee, T. (2019). Recent Progress in Inkjet-Printed Thin-Film Transistors. Adv. Sci. 6 (6), 1801445. doi:10.1002/advs.201801445

Faasen, D. P., Jarray, A., Zandvliet, H. J. W., Kooij, E. S., and Kwiecinski, W. (2020). Hansen Solubility Parameters Obtained via Molecular Dynamics Simulations as a Route to Predict Siloxane Surfactant Adsorption. J. Colloid Interf. Sci. 575 (September), 326-336. doi:10.1016/j.jcis.2020.04.070

Fang, Y., Hester, J. G. D., deGlee, B. M., Tuan, C.-C., Brooke, P. D., Le, T., et al. (2016a). A Novel, Facile, Layer-By-Layer Substrate Surface Modification for the Fabrication of All-Inkjet-Printed Flexible Electronic Devices on Kapton. J. Mater. Chem. C. 4 (29), 7052-7060. doi:10.1039/C6TC01066K

Fang, Y., Hester, J. G. D., Su, W., Chow, J. H., Sitaraman, S. K., and Tentzeris, M. M. (2016b). A Bio-Enabled Maximally Mild Layer-By-Layer Kapton Surface Modification Approach for the Fabrication of All-Inkjet-Printed Flexible Electronic Devices. Sci. Rep. 6 (1), 39909. doi:10.1038/srep39909

Gao, L., Cao, K., Hu, X., Xiao, R., Gan, B., Wang, W., et al. (2020). Nano Electromechanical Approach for Flexible Piezoresistive Sensor. Appl. Mater. Today. 18 (March), 100475. doi:10.1016/j.apmt.2019.100475

Gao, L., Han, Y., Surjadi, J. U., Cao, K., Zhou, W., Xu, H., et al. (2021a). Magnetically Induced Micropillar Arrays for an Ultrasensitive Flexible Sensor With a Wireless Recharging System. Sci. China Mater. 64 (8), 1977-1988. doi:10.1007/s40843-020-1637-9

Gao, L., Wang, M., Wang, W., Xu, H., Wang, Y., Zhao, H., et al. (2021b). Highly Sensitive Pseudocapacitive Iontronic Pressure Sensor With Broad Sensing Range. Nano-micro Lett. 13 (1), 140. doi:10.1007/s40820-021-00664-w

Gao, L., Wang, Y., Hu, X., Zhou, W., Cao, K., Wang, Y., et al. (2019). Cellular Carbon-Film-Based Flexible Sensor and Waterproof Supercapacitors. ACS Appl. Mater. Inter. 11 (29), 26288-26297. doi:10.1021/acsami.9b09438

Ghosh, I., Konar, J., and Bhowmick, A. K. (1997). Surface Properties of Chemically Modified Polyimide Films. J. Adhes. Sci. Technology. 11 (6), 877-893. doi:10.1163/156856197X00967

Grandoni, A., Mannini, G., Glisenti, A., Manariti, A., and Galli, G. (2017). Use of Statistical Design of Experiments for Surface Modification of Kapton Films by CF 4 O 2 Microwave Plasma Treatment. Appl. Surf. Sci. 420 (October), 579-585. doi:10.1016/j.apsusc.2017.05.140
Research Funds for the Central Universities (JB210407), the Youth Innovation Promotion Association CAS (no. 2021363), and the SIAT Innovation Program for Excellent Young Researchers (no. 201803). Shenzhen Science and Technology Program (JCYJ20210324102008023).

\section{SUPPLEMENTARY MATERIAL}

The Supplementary Material for this article can be found online at: https://www.frontiersin.org/articles/10.3389/fmats.2021.769071/ full\#supplementary-material

Granqvist, C. G. (2007). Transparent Conductors as Solar Energy Materials: A Panoramic Review. Solar Energ. Mater. Solar Cell 91 (17), 1529-1598. doi:10.1016/j.solmat.2007.04.031

Han, T.-H., Lee, Y., Choi, M.-R., Woo, S.-H., Bae, S.-H., Hong, B. H., et al. (2012). Extremely Efficient Flexible Organic Light-Emitting Diodes With Modified Graphene Anode. Nat. Photon. 6 (2), 105-110. doi:10.1038/nphoton.2011.318

He, P., Cao, J., Ding, H., Liu, C., Neilson, J., Li, Z., et al. (2019). Screen-Printing of a Highly Conductive Graphene Ink for Flexible Printed Electronics. ACS Appl. Mater. Inter. 11 (35), 32225-32234. doi:10.1021/acsami.9b04589

Huang, X. D., Bhangale, S. M., Moran, P. M., Yakovlev, N. L., and Pan, J. (2003). Surface Modification Studies of KaptonHN Polyimide Films. Polym. Int. 52 (7), 1064-1069. doi:10.1002/pi.1143

Huang, X., Liu, Y., Cheng, H., Shin, W.-J., Fan, J. A., Liu, Z., et al. (2014). Materials and Designs for Wireless Epidermal Sensors of Hydration and Strain. Adv. Funct. Mater. 24 (25), 3846-3854. doi:10.1002/adfm.201303886

Inagaki, N., Tasaka, S., and Hibi, K. (1992). Surface Modification of Kapton Film by Plasma Treatments. J. Polym. Sci. A. Polym. Chem. 30 (7), 1425-1431. doi:10.1002/pola.1992.080300722

Jarray, A., Wijshoff, H., Luiken, J. A., and den Otterden Otter, W. K. (2020). Systematic Approach for Wettability Prediction Using Molecular Dynamics Simulations. Soft Matter. 16 (17), 4299-4310. doi:10.1039/D0SM00197J

Kim, S.-Y., Kim, K., Hwang, Y. H., Park, J., Jang, J., Nam, Y., et al. (2016). HighResolution Electrohydrodynamic Inkjet Printing of Stretchable Metal Oxide Semiconductor Transistors With High Performance. Nanoscale. 8 (39), 17113-17121. doi:10.1039/C6NR05577J

Kondo, T., Watanabe, R., Shimoyama, Y., Shinohe, K., Kulinich, S. A., and Iwamori, S. (2017). Effect of Reactive Oxygen Species Generated with Ultraviolet Lamp and Plasma on Polyimide Surface Modification. Surf Interf. Anal. 49 (11), 1069-1077. doi:10.1002/sia.6279

Lai, F., Sun, Z., Saji, S. E., He, Y., Yu, X., Zhao, H., et al. (2021). Machine LearningAided Crystal Facet Rational Design with Ionic Liquid Controllable Synthesis. Small. 17 (12), 2100024. doi:10.1002/smll.202100024

Least, B. T., and Willis, D. A. (2013). Modification of Polyimide Wetting Properties by Laser Ablated Conical Microstructures. Appl. Surf. Sci. 273 (May), 1-11. doi:10.1016/j.apsusc.2012.12.141

Li, J., Sollami Delekta, S., Zhang, P., Yang, S., Lohe, M. R., Zhuang, X., et al. (2017). Scalable Fabrication and Integration of Graphene Microsupercapacitors through Full Inkjet Printing. ACS Nano. 11 (8), 8249-8256. doi:10.1021/ acsnano.7b03354

Li, J., Ye, F., Vaziri, S., Muhammed, M., Lemme, M. C., and Östling, M. (2013). Efficient Inkjet Printing of Graphene. Adv. Mater. 25 (29), 3985-3992. doi:10.1002/adma.201300361

Li, X., Wang, W., Wu, L., Zhao, H., Wang, M., Wang, Y., et al. (2021). Wearable, Self-Cleaning, Wireless Integrated Tactile Sensory System With Superior Sensitivity. Sensors Actuators A: Phys. 331 (November), 113027. doi:10.1016/ j.sna.2021.113027

Lien, D.-H., Kao, Z.-K., Huang, T.-H., Liao, Y.-C., Lee, S.-C., and He, J.-H. (2014). All-Printed Paper Memory. ACS Nano. 8 (8), 7613-7619. doi:10.1021/ nn501231z

Liu, F., Hu, N., Ning, H., Liu, Y., Li, Y., and Wu, L. (2015). Molecular Dynamics Simulation on Interfacial Mechanical Properties of Polymer Nanocomposites 
With Wrinkled Graphene. Comput. Mater. Sci. 108 (October), 160-167. doi:10.1016/j.commatsci.2015.06.023

Liu, Y., Yiu, C., Jia, H., Wong, T., Yao, K., Huang, Y., et al. (2021). Thin, Soft, Garment-integrated Triboelectric Nanogenerators for Energy Harvesting and Human Machine Interfaces. EcoMat. 3 (4), e12123. doi:10.1002/eom2.12123

Lu, P., Wang, L., Zhu, P., Huang, J., Wang, Y., Bai, N., et al. (2021). Iontronic Pressure Sensor With High Sensitivity and Linear Response Over a Wide Pressure Range Based on Soft Micropillared Electrodes. Sci. Bull. 66 (11), 1091-1100. doi:10.1016/j.scib.2021.02.019

Marques, A. C., Faria, J., Perdigão, P., Faustino, B. M. M., Ritasalo, R., Costabello, K., et al. (2019). Stability under Humidity, UV-Light and Bending of AZO Films Deposited by ALD on Kapton. Sci. Rep. 9 (1), 17919. doi:10.1038/s41598-01954451-0

Minemawari, H., Yamada, T., Matsui, H., Tsutsumi, J. y., Haas, S., Chiba, R., et al. (2011). Inkjet Printing of Single-Crystal Films. Nature. 475 (7356), 364-367. doi:10.1038/nature10313

Mizukami, M., Cho, S.-I., Watanabe, K., Abiko, M., Suzuri, Y., Tokito, S., et al. (2018). Flexible Organic Light-Emitting Diode Displays Driven by InkjetPrinted High-Mobility Organic Thin-Film Transistors. IEEE Electron. Device Lett. 39 (1), 39-42. doi:10.1109/LED.2017.2776296

Muraru, S., Burns, J. S., and Ionita, M. (2020). GOPY: A Tool for Building 2D Graphene-Based Computational Models. SoftwareX. 12 (July), 100586. doi:10.1016/j.softx.2020.100586

Moses, O. A., Gao, L., Zhao, H., Wang, Z., Adam, M. L., and Sun, Z. (2021). 2D Materials Inks toward Smart Flexible Electronics. Mater. Today. doi:10.1016/ j.mattod.2021.08.010

Noguchi, Y., Sekitani, T., Yokota, T., and Someya, T. (2008). Direct Inkjet Printing of Silver Electrodes on Organic Semiconductors for Thin-Film Transistors With Top Contact Geometry. Appl. Phys. Lett. 93 (4), 043303. doi:10.1063/ 1.2959728

Park, J. B., Oh, J. S., Gil, E. L., Kyoung, S. J., Lim, J. T., and Yeom, G. Y. (2010). Polyimide Surface Treatment by Atmospheric Pressure Plasma for Metal Adhesion. J. Electrochem. Soc. 157 (12), D614. doi:10.1149/1.3493585

Park, S.-J., Sohn, H.-J., Hong, S.-K., and Shin, G.-S. (2009). Influence of Atmospheric Fluorine Plasma Treatment on Thermal and Dielectric Properties of Polyimide Film. J. Colloid Interf. Sci. 332 (1), 246-250. doi:10.1016/j.jcis.2008.12.040

Patil, S. U., Radue, M. S., Pisani, W. A., Deshpande, P., Xu, H., Al Mahmud, H., et al. (2020). Interfacial Characteristics between Flattened CNT Stacks and Polyimides: A Molecular Dynamics Study. Comput. Mater. Sci. 185 (December), 109970. doi:10.1016/j.commatsci.2020.109970

Peng, X., Yuan, J., Shen, S., Gao, M., Chesman, A. S. R., Yin, H., et al. (2017). Perovskite and Organic Solar Cells Fabricated by Inkjet Printing: Progress and Prospects. Adv. Funct. Mater. 27 (41), 1703704. doi:10.1002/adfm.201703704

Plimpton, S. (1995). Fast Parallel Algorithms for Short-Range Molecular Dynamics. J. Comput. Phys. 117 (1), 1-19. doi:10.1006/jcph.1995.1039

Rusu, R.-D., Constantin, C.-P., Drobota, M., Gradinaru, L.-M., Butnaru, M., and Pislaru, M. (2020). Polyimide Films Tailored by UV Irradiation: Surface Evaluation and Structure-Properties Relationship. Polym. Degrad. Stab. 177 (July), 109182. doi:10.1016/j.polymdegradstab.2020.109182

Saidina, D. S., Eawwiboonthanakit, N., Mariatti, M., Fontana, S., and Hérold, C. (2019). Recent Development of Graphene-Based Ink and Other Conductive Material-Based Inks for Flexible Electronics. J. Elec Materi. 48 (6), 3428-3450. doi:10.1007/s11664-019-07183-w
Sekitani, T., Yokota, T., Zschieschang, U., Klauk, H., Bauer, S., Takeuchi, K., et al. (2009). Organic Nonvolatile Memory Transistors for Flexible Sensor Arrays. Science. 326 (5959), 1516-1519. doi:10.1126/science.1179963

Shin, J.-W., Jeun, J.-P., and Kang, P.-H. (2010). Surface Modification and Characterization of $\mathrm{N}+$ Ion Implantation on Polyimide Film. Macromol. Res. 18 (3), 227-232. doi:10.1007/s13233-010-0310-x

Song, D., Mahajan, A., Secor, E. B., Hersam, M. C., Francis, L. F., and Frisbie, C. D. (2017). High-Resolution Transfer Printing of Graphene Lines for Fully Printed, Flexible Electronics. ACS Nano. 11 (7), 7431-7439. doi:10.1021/ acsnano.7b03795

Templier, F., Aventurier, B., Demars, P., Botrel, J.-L., and Martin, P. (2007). Fabrication of High Performance Low Temperature Poly-Silicon Backplanes on Metal Foil for Flexible Active-Matrix Organic Light Emission Diode Displays. Thin Solid Films. 515 (19), 7428-7432. doi:10.1016/j.tsf.2006.11.197

Usami, K., Ishijima, T., and Toyoda, H. (2012). Rapid Plasma Treatment of Polyimide for Improved Adhesive and Durable Copper Film Deposition. Thin Solid Films. 521 (October), 22-26. doi:10.1016/j.tsf.2012.03.080

Xie, J., Xin, D., Cao, H., Wang, C., Zhao, Y., Yao, L., et al. (2011). Improving Carbon Fiber Adhesion to Polyimide With Atmospheric Pressure Plasma Treatment. Surf. Coat. Technology. 206 (2), 191-201. doi:10.1016/ j.surfcoat.2011.04.016

Xu, H., Gao, L., Wang, Y., Cao, K., Hu, X., Wang, L., et al. (2020). Flexible Waterproof Piezoresistive Pressure Sensors With Wide Linear Working Range Based on Conductive Fabrics. Nano-micro Lett. 12 (1), 159. doi:10.1007/ s40820-020-00498-y

Yang, S., Gao, F., and Qu, J. (2013). A Molecular Dynamics Study of Tensile Strength Between a Highly-Crosslinked Epoxy Molding Compound and a Copper Substrate. Polymer. 54 (18), 5064-5074. doi:10.1016/ j.polymer.2013.07.019

Zhou, L., Wanga, A., Wu, S.-C., Sun, J., Park, S., and Jackson, T. N. (2006). AllOrganic Active Matrix Flexible Display. Appl. Phys. Lett. 88 (8), 083502. doi:10.1063/1.2178213

Zhou, P., Liao, Y., Li, Y., Pan, D., Cao, W., Yang, X., et al. (2019). An Inkjet-Printed, Flexible, Ultra-Broadband Nanocomposite Film Sensor for In-Situ Acquisition of High-Frequency Dynamic Strains. Composites A: Appl. Sci. Manufacturing. 125 (October), 105554. doi:10.1016/j.compositesa.2019.105554

Conflict of Interest: The authors declare that the research was conducted in the absence of any commercial or financial relationships that could be construed as a potential conflict of interest.

Publisher's Note: All claims expressed in this article are solely those of the authors and do not necessarily represent those of their affiliated organizations, or those of the publisher, the editors and the reviewers. Any product that may be evaluated in this article, or claim that may be made by its manufacturer, is not guaranteed or endorsed by the publisher.

Copyright () $2021 \mathrm{Wu}$, Wang, Zhao, Gao, Lu and Sun. This is an open-access article distributed under the terms of the Creative Commons Attribution License (CC BY). The use, distribution or reproduction in other forums is permitted, provided the original author(s) and the copyright owner(s) are credited and that the original publication in this journal is cited, in accordance with accepted academic practice. No use, distribution or reproduction is permitted which does not comply with these terms. 